\title{
Characteristics of rifampicin-resistant tuberculosis detection in China, 2015-2019
}

\author{
Wei Su, Yun-zhou Ruan, Tao Li, Xin Du, Jia-wen Jiang and Ren-zhong Li (ID $^{*}$
}

\begin{abstract}
Background: The very high burden of rifampicin resistance tuberculosis (RR-TB) and the very low detection of RR-TB cases are a major challenge that China has been facing. This study analyzed the characteristics of RR-TB detection in China after the change of RR-TB detection strategy since 2015, aiming to provide reference and evidence for the development of more precise national drug resistance tuberculosis prevention and control policy.

Methods: We extracted data related to rifampicin resistance screening from the national Tuberculosis Information Management System (TBIMS) from 2015 to 2019, and used descriptive research methods to analyze the screening rate of presumptive RR-TB, the number and duration of RR-TB patients detected and drug resistance testing methods in each year. Chi-square test was used to compare the differences in component ratio or rate between years, and Kruskal Wallis test was used to compare the differences in median days for detection of RR-TB patients in each year.
\end{abstract}

Results: A total of 68,200 RR-TB cases were detected during 2015-2019, of which 48.1\% were new cases. The number and detection rate of RR-TB cases increased year by year, from 10019 and 14.3\% in 2015 to 18623 and 28.7\% in 2019, respectively. Of the bacteriologically confirmed TB cases, $81.9 \%$ were tested for RR in 2019, a considerable increase from 29.5\% in 2015. In 2019, only 41.0\% of RR-TB cases had fluoroquinolones (FQs) susceptibility testing performed, and this proportion has been declining year by year since 2016. The proportion of application of rapid molecular tools increased from $24.0 \%$ in 2015 to $67.1 \%$ in 2019, and the median days to obtain RR results was significantly shortened. In 2019, 76.0\% of RR-TB cases were diagnosed as presumptive RR-TB in county-level hospitals.

Conclusions: After China modified the RR-TB detection strategy, the screening rate of RR and the number of RR-TB cases increased significantly. The RR testing methods now predominantly utilize rapid molecular tools. However, comprehensive measures should be implemented to close the gap in the detection of RR-TB cases. It is imperative to take FQs susceptibility testing seriously and effectively strengthen the laboratory capacity of county-level hospitals.

Keywords: Rifampicin-resistant tuberculosis, Case detection, Detection policy, China

\section{Background}

Drug-resistant tuberculosis (TB) remains an important public health concern worldwide, especially rifampicin resistance TB (RR-TB). RR-TB is defined as any resistance to rifampicin, including mono-resistance, multidrug resistance, polydrug resistance [1]. In 2019, there were

*Correspondence: lirz@chinacdc.cn

National Center for Tuberculosis Control and Prevention, China CDC, No.155 Changbai Road, Changping District, Beijing 102206, People's Republic of China
465000 RR-TB cases worldwide; $56 \%$ were undetected. Even when the diagnosis was made, only $57 \%$ achieved a successful treatment outcome [2].

China is a country with a high burden of RR-TB. The number of cases in China accounts for $14 \%$ of global RR-TB cases, ranking second after India. The rifampicin resistance (RR) rates of new and retreated TB cases were $7.1 \%$ and $23.0 \%$, respectively, which were higher than the global levels of $3.3 \%$ and $17.7 \%$ [2]. China initiated the Programmatic Management of Drug-resistant Tuberculosis (PMDT) in 2006 with the support of the Global 
Fund Project. By the end of June 2014, when the Global Fund Project closed in China, nearly one-third of the prefectures in 30 provinces (China has 31 provinces) had implemented PMDT [3, 4]. However, due to limited resources and personnel capabilities, traditional phenotypic drug susceptibility testing (DST) was mainly used at this stage and only the drug susceptibility of RR high-risk groups was tested. Not only did it take longer to diagnose patients, but fewer RR-TB patients were detected [5]. In 2012-2014, China only detected $5.0 \%, 7.7 \%$, and $11.3 \%$, respectively, of RR-TB cases estimated by the World Health Organization (WHO) [6-8]. The very high burden of RR-TB and the very low RR-TB case detection had been the major challenge facing China since the implementation of PMDT in 2006.

WHO's End TB Strategy calls for the early diagnosis of TB and for universal drug-susceptibility testing [9]. In order to achieve the goal of End TB Strategy and solve the issue of lower detection of RR-TB cases, when the Global Fund Project ended and since 2015 China has modified the RR-TB detection strategy to scale up drug resistance screening from only high-risk groups of RR-TB to all bacteriologically confirmed TB cases. In particular, the "13th Five-Year National Tuberculosis Prevention and Control Program (2016-2020)" (13th Five-Year TB Program) adopted the expansion strategy of RR-TB detection and called for the priority application of rapid molecular technology for drug susceptibility testing to shorten the diagnosis time. It is required that the screening rate of high-risk groups for RR reach $95 \%$ and achieve full coverage of PMDT by the end of the 13th Five-Year Plan in 2020 [10]. In order to fulfill the commitment of the political declaration of the first United Nations (UN) High-Level Conference on Tuberculosis held in 2018 [11], the Chinese government issued the "Stop Tuberculosis Action Plan (2019-2022)" in 2019 and set a more ambitious target for rifampicin resistance testing. The new target is to have $90 \%$ of bacteriologically confirmed TB cases screened for rifampicin resistance by 2022 [12].

Benefiting from the accumulated experience during the Global Fund Project and aggressive promotion of the national 13th Five-Year TB Program and the Stop TB Action Plan (2019-2022), the number of RR-TB cases detected in China has increased significantly every year since 2015. In order to better understand the changes of RR-TB detection in China after 2015 and provide evidence for the national drug-resistant TB prevention and control strategy, we present a review of the current status of RR-TB detection in China from 2015 to 2019 based on data of the national Tuberculosis Information Management System (TBIMS) [13]. This review focuses on the RR screening rate, the number of RR-TB cases detected, and the application of rifampicin resistance diagnostic tools as well as the change in the source of presumptive RR-TB cases. Since fluoroquinolones (FQs) are another core anti-tuberculosis drug besides rifampicin, whether there is resistance to FQs has a great impact on the choice of chemotherapy regimens and treatment outcomes $[14,15]$. Moreover, WHO defines at least $80 \%$ of bacteriologically confirmed TB patients undergoing rifampicin resistance testing and at least $80 \%$ of RR-TB patients undergoing FQs resistance testing as good testing coverage [2]. Therefore, we also analyzed fluoroquinolones resistance (FQR) among RR-TB patients. This study aims to provide reference and evidence for the development of more precise national drug resistance tuberculosis prevention and control strategy in the future.

\section{Methods}

\section{Data sources}

The data was extracted from the national TBIMS. The bacteriologically confirmed TB cases notified in the TBIMS and of which were diagnosed with RR-TB cases were included in our analysis from January 1, 2015 to December 31, 2019. Only tuberculosis-related information in TBIMS was used, and confidential information such as patients' personal identity was not involved.

\section{DST methods and procedure}

China currently applies rapid molecular and traditional phenotypic diagnostic tools to test the susceptibility of anti-tuberculosis drugs. Rapid molecular assays include Xpert MTB/RIF (Xpert) and line probe assays (LPAs), which are recommended by the WHO [16], as well as domestically produced MeltPro TB assays (MeltPro) [17] and Genechip [18]. MeltPro can detect isoniazid, rifampicin, and FQ resistance. Genechip can detect isoniazid and rifampicin resistance. Bacteriologically confirmed TB cases are the subjects of DST, and at least rifampicin, isoniazid, $\mathrm{FQ}$, and second-line injections are tested for drug susceptibility. If rapid molecular methods available, rapid DST will be preferred. In the implementation of DST, the identification of Mycobacterium tuberculosis complex and non-tuberculous mycobacteria (NTM) was carried out. The current rifampicin resistance has ruled out NTM infection.

The DST procedure is first to determine whether a presumptive TB patient is bacteriologically confirmed and then perform DST on the bacteriologically confirmed TB patient using molecular or traditional testing tools. Specifically, county-level TB designated hospitals diagnose bacteriologically confirmed TB cases, of which counties equipped with Xpert conduct rapid RR testing for bacteriologically confirmed TB cases. The confirmed RR-TB cases will be referred to the prefecture-level designated TB hospitals for DST of other drugs; those counties 
without Xpert need to transport sputum smear-positive specimens or culture-positive strains to the prefecturelevel TB designated hospitals for DST of rifampicin and other drugs.

\section{Laboratory quality control}

China has established a complete laboratory network system at the national, provincial, prefecture and county levels and has a sound quality assurance (QA) system [19]. Laboratories at all levels not only carry out quality control (QC) but also accept external quality assessments (EQA). All laboratories are qualified to carry out testing only after passing quality assessment. Laboratories that carry out DST need to undergo a proficiency test organized by the National Tuberculosis Reference Laboratory once a year. County-level laboratories that carry out sputum smear microscopy need to undergo blind re-examination by prefecture-level laboratories every quarter.

\section{Definitions}

A bacteriologically confirmed TB case refers to sputum smear positive, only culture positive, or only positive molecular testing positive [20]. The presumptive RR-TB patient in this study refers to a TB patient who is bacteriologically confirmed. High-risk groups refer to at least one of the following: (a) chronic TB patients/failure of retreatment TB patients, (b) close contact with a known RR-TB patient, (c) new TB patients of initial treatment failure, (d) relapsed or returned TB patients or (e) new TB patients remaining sputum culture or smear positive at the end of the 2nd month after treatment [21].

\section{Statistical analysis}

Relevant data was derived from TBIMS and a descriptive analysis method was used to compare the changes of RR-TB cases detection in 2015-2019. The enumeration data was described by component ratio or rate. The comparison of inter-annual differences was tested by
Chi-squared test. The measurement data was presented by medians with an interquartile range (IQR), and the comparison of inter-annual differences was tested by Kruskal Wallis test. All $P$ values are two-tailed; a value less than 0.05 was considered statistically significant. All statistical analyses were done with SPSS software version 20.0 (SPSS Inc., Chicago, IL, USA).

\section{Results}

The status of RR-TB detection and FQs resistance testing A total of 68200 patients were detected in 2015-2019, of which $48.1 \%$ were new patients. Only $50.3 \%$ of all RR-TB patients were tested for FQ susceptibility. The number of RR-TB patients detected, the RR screening rate, and the coverage rate of PMDT increased year by year. However, during 2015-2017, the first 3 years when the RR-TB case detection strategy changed, the rising trend was not obvious and the RR screening rate even declined slightly in 2016. Since 2018, in the late period of the 13th Five-Year TB Program, there has been a significant increase. By 2019, the number of patients detected, the RR screening rate, and the coverage rate of PMDT reached the highest level, 1.9 times (18 623/10 019), 2.8 times (81.9\%/29.5\%) and 1.4 times (95/70) that of 2015 respectively. The proportion of FQ susceptibility testing in RR-TB cases had been declining year by year since 2016, and was the lowest in 2019 (Table 1).

\section{Comparison with the number of RR-TB patients estimated by the WHO}

The rate of bacteriologically confirmed TB cases increased significantly from $31 \%$ in 2015 to $47 \%$ in 2019 . At the same time, the number of RR-TB cases increased year by year. Comparing the number of detected RR-TB cases with the WHO estimate, it can be found that the gap narrowed year by year. The annual detection rates of RR-TB cases were 14.3\%, 15.6\%, 17.8\%, 22.8\% and 28.7\% respectively (Fig. 1).

Table 1 The status of RR-TB detection, FQs resistance testing and PMDT coverage in China, 2015-2019

\begin{tabular}{|c|c|c|c|c|c|c|c|c|c|c|}
\hline \multirow[t]{2}{*}{ Year } & \multicolumn{3}{|c|}{ RR-TB cases detected } & \multicolumn{3}{|c|}{ RR screening rate (\%) } & \multicolumn{3}{|c|}{$\begin{array}{l}\text { Proportion of FQs screening in } \\
\text { RR-TB cases }(\%)\end{array}$} & \multirow{2}{*}{$\begin{array}{l}\text { Prefecture-level } \\
\text { coverage rate for } \\
\text { PMDT (\%) }\end{array}$} \\
\hline & All & $\begin{array}{l}\text { New cases } \\
n(\%)\end{array}$ & $\begin{array}{l}\text { High-risk groups } \\
n(\%)\end{array}$ & All & New cases & $\begin{array}{l}\text { High-risk } \\
\text { groups }\end{array}$ & All & New cases & $\begin{array}{l}\text { High-risk } \\
\text { groups }\end{array}$ & \\
\hline 2015 & 10019 & 3971 (39.6) & $6048(60.4)$ & 29.5 & 23.1 & 57.3 & 57.8 & 58.6 & 57.2 & 70.0 \\
\hline 2016 & 11423 & $4564(40.0)$ & $6859(60.0)$ & 28.6 & 22.8 & 54.8 & 59.6 & 60.3 & 59.0 & 70.0 \\
\hline 2017 & 13069 & $6227(47.6)$ & $6842(52.4)$ & 37.8 & 33.1 & 57.2 & 51.9 & 52.7 & 51.1 & 78.0 \\
\hline 2018 & 15066 & 7807 (51.8) & $7259(48.2)$ & 62.8 & 60.2 & 72.6 & 48.2 & 51.3 & 45.0 & 90.0 \\
\hline 2019 & 18623 & $10204(54.8)$ & $8419(45.2)$ & 81.9 & 80.4 & 88.4 & 41.0 & 42.7 & 39.0 & 95.0 \\
\hline Total & 68200 & 32773 (48.1) & 35427 (51.9) & 49.4 & 45.3 & 67.2 & 50.3 & 51.0 & 49.6 & - \\
\hline
\end{tabular}




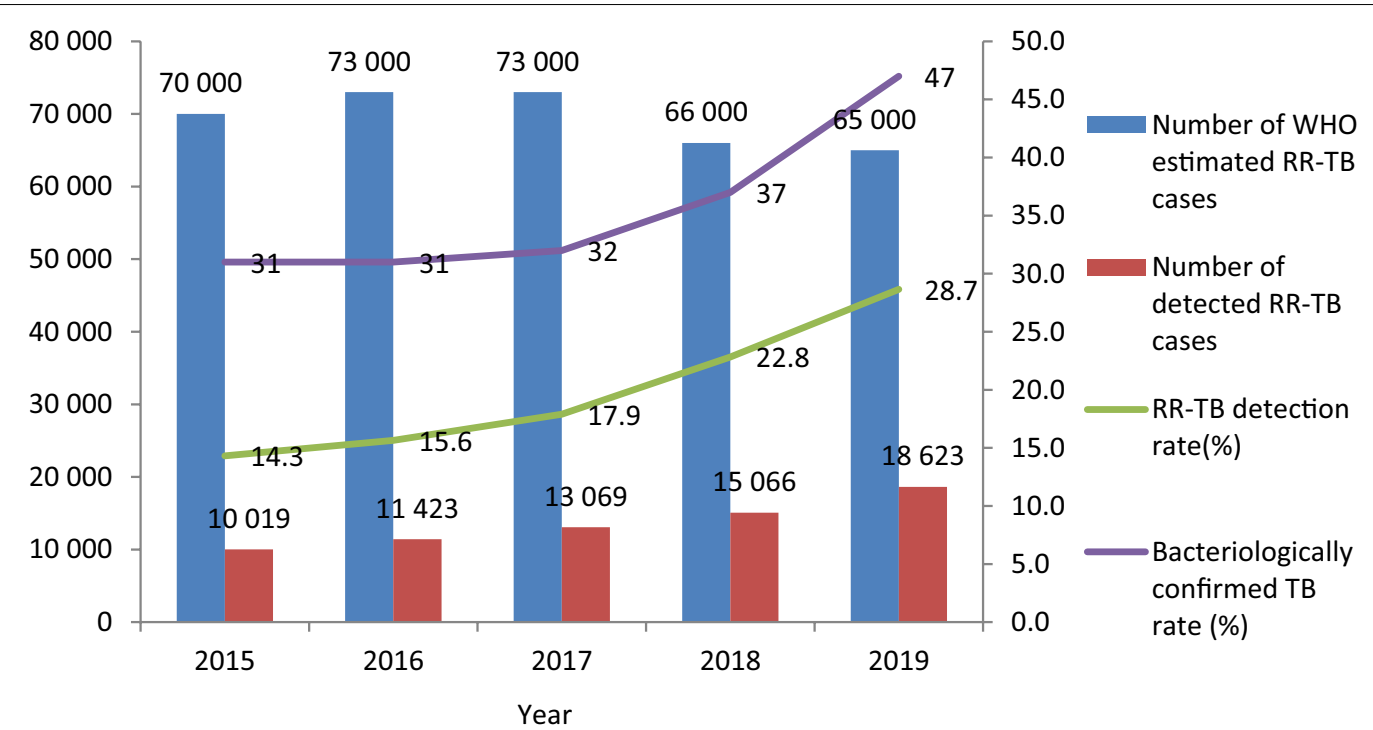

Fig. 1 Comparison between the number of RR-TB cases detected and the WHO estimated, 2015-2019. Number of WHO estimated RR-TB cases, bacteriologically confirmed TB rate: from annual WHO TB report. RR-TB Rifampicin resistance tuberculosis

\section{DST methods and time to RR results notification}

From 2015 to 2019, 52.2\% of all patients used traditional phenotypic DST, which was slightly higher than that of rapid DST (47.8\%). Although traditional DST was the main method, the application of rapid DST increased year by year, from $24.0 \%$ in 2015 to $67.1 \%$ in 2019 , and the proportion of rapid DST has exceeded that of traditional DST since 2018. In 2019, the proportion of RR detected by rapid DST was $67.1 \%$, which was 2.0 times that of the traditional method $(67.1 \% / 32.9 \%)$. The changes in the proportion of applying rapid DST annually were significant (Chi-square trend test, $P<0.0001$ ) (Table 2).

The time interval from the sputum smear results notification to the obtaining of rifampin susceptibility results reduced year by year. From 2015 to 2019, the median time was 61 days (IQR: 27-91), 52 days (IQR: 21-86), 44 days (IQR: 9-81), 34 days (IQR: 6-72), and
15 days (IQR: 2-55) respectively. There was a significant difference in the time of obtaining DST results in each year (Kruskal Wallis test, $P<0.0001$ ) (Fig. 2).

\section{Change in the source of presumptive RR-TB cases}

In 2015-2019, the source of presumptive RR-TB cases showed different changes at different stages. During 2015-2017, the proportion of RR-TB cases diagnosed as presumptive RR-TB in county-level TB designated hospitals decreased slightly, from $62.1 \%$ in 2015 to $57.2 \%$ in 2017. But from 2018, this trend has reversed and showed a sharp rise, reaching a maximum of $76 \%$ in 2019. The change in the source of presumptive RR-TB annually was significant (Chi-squared test, $P<0.0001$ ) (Table 2).

Table 2 DST method and the source of presumptive RR-TB cases

\begin{tabular}{|c|c|c|c|c|c|c|c|}
\hline & $\begin{array}{l}2015 \\
(n=10019)\end{array}$ & $\begin{array}{l}2016 \\
(n=11423)\end{array}$ & $\begin{array}{l}2017 \\
(n=13069)\end{array}$ & $\begin{array}{l}2018 \\
(n=15066)\end{array}$ & $\begin{array}{l}2019 \\
(n=18623)\end{array}$ & $\begin{array}{l}\text { Total } \\
(n=68200)\end{array}$ & $P$ value \\
\hline \multicolumn{8}{|l|}{ DST method } \\
\hline Rapid & $2407(24.0)$ & $3714(32.5)$ & $5623(43.0)$ & $8378(55.6)$ & $12501(67.1)$ & $32623(47.8)$ & 0.000 \\
\hline Traditional & $7612(76.0)$ & 7709 (67.5) & $7445(57.0)$ & $6688(44.4)$ & $6122(32.9)$ & $35577(52.2)$ & \\
\hline \multicolumn{8}{|c|}{ The source of presumptive RR-TB cases } \\
\hline County-level hospital & $6224(62.1)$ & $6722(58.8)$ & $7469(57.2)$ & $11133(73.9)$ & $14155(76.0)$ & $45703(67.0)$ & 0.000 \\
\hline $\begin{array}{l}\text { Prefecture-level and } \\
\text { above hospitals }\end{array}$ & $3795(37.9)$ & $4701(41.2)$ & $5600(42.8)$ & $3933(26.1)$ & $4468(24.0)$ & $22497(33.0)$ & \\
\hline
\end{tabular}

Data are presented as $n$ (\%)

DST Drug susceptibility testing, RR-TB Rifampicin-resistant tuberculosis 


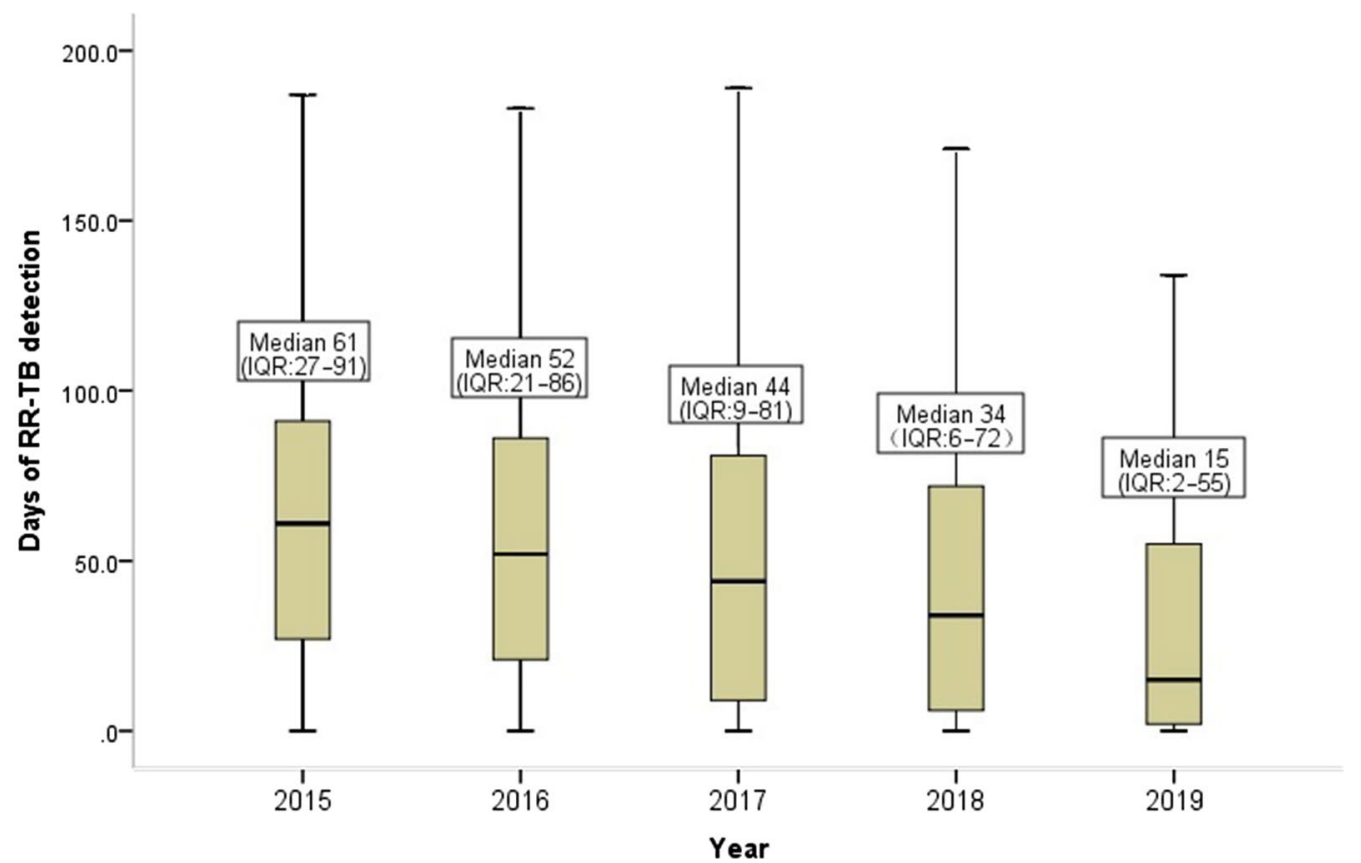

Fig. 2 Days of RR-TB diagnosis: the time interval from the reporting time of sputum smear results to the reporting time of rifampicin susceptibility results. RR-TB Rifampicin resistance tuberculosis

\section{Discussion}

This study analyzed the characteristics of RR-TB case detection at the level of PMDT after 2015, especially after implementation of the national 13th Five-Year TB Program in 2016 in China.

Our analysis indicated that the RR screening rate and the number of RR-TB cases detection have increased significantly from 2015 to 2020 . In 2019, the screening rate of RR in all bacteriologically confirmed TB cases reached $81.9 \%$, exceeding the global level of $61 \%$ [2], and has become a routine work of the National TB Program (NTP). Delay in the diagnosis of RR-TB is a significant cause for the death, loss, and inappropriate treatment for RR-TB cases [22-24], as well as increases in the risk of drug resistance spreading in the community. In 2019 the median time for RR-TB cases to obtain RR results have been significantly reduced to 15 days, which is lower than the 26 days in the Republic of Korea [25]. In Shanghai, China, a metropolis with a population of 30 million, the diagnosis time has been as short as 9 days [26].

The main reason for the above changes is that after the national drug resistant cases detection policy was modified, in order to achieve the national 13th Five-Year TB Program targets the governments at all levels strengthened their commitment, especially near the end of the 13th Five-Year TB Program. This was done by increasing the capabilities of laboratories in weak areas and the aggressive scale up of the use of rapid molecular DST tools. From 2015 to 2018, the Chinese government has invested a total of about 260 million CNY to support economically underdeveloped areas such as the central and western regions to equip rapid molecular diagnostic tools [27]. Furthermore, China's central government provided funds for the transportation costs of the county-level sputum specimens and strains and raised funds from various sources to provide free screening for presumptive RR-TB cases. These comprehensive measures improved the detection of RR-TB. Although the RR screening rate among high-risk groups has not yet reached the target of the 13th Five-Year TB Program, it will be achievable given the right strategic focus complemented by sustained leadership and adequate resources in 2020.

Our analysis also revealed that the source of presumptive RR-TB cases has changed in 2015-2019. More and more presumptive RR-TB cases are diagnosed in countylevel hospitals, and the proportion is as high as $76.0 \%$ in 2019. The main reason for this trend is that the 13th Five-Year TB Program called for improving TB graded diagnosis and treatment services in order to strengthen the integration of TB prevention and treatment and make more rational use of medical resources [10]. By enhancing the capacity of county-level TB designated hospitals and the differentiation of medical insurance reimbursement to guide patients visiting hospitals in their jurisdiction to improve the accessibility of health care. This finding is of significance to China's future deployment of 
PMDT. Although the diagnosis of drug-resistant TB is set at the prefecture level, it is necessary to include countylevel rapid rifampicin susceptibility testing so that RR cases can receive appropriate treatment early and thus reduce the spread of drug resistance. Only by improving the TB diagnosis capabilities of county-level hospitals "missing RR-TB cases" can be reduced.

Our analysis also found some concerning issues. First, the proportion of new RR-TB cases has reached 54.8\% in 2019. New RR-TB cases represent primary rifampicin resistance, suggesting that we need to pay attention to the spread of drug-resistant tuberculosis. Some studies in China have shown the transmission of multidrug-resistant tuberculosis $[28,29]$. Our analysis results also indicate that the potential risk of community transmission of RR-TB in China is relatively high in recent years. China currently has no legislation on isolation treatment or travel restrictions for infectious RR-TB cases. It is imperative that China explores the possibility of legislation to manage infectious RR-TB in the future.

A second key concern is the continued low detection rate of RR-TB cases. 2019 was the year with the largest number of cases diagnosed, but only $28 \%$ of the RR-TB patients estimated by WHO were detected. This suggests that more than $70 \%$ of RR-TB cases were being missed. The main reasons are, first, that the rate of bacteriologically confirmed TB cases in China was low. It was $47 \%$ in 2019, which is lower than the global rate of 57\% [2]. Second, $5 \%$ of prefectures nationwide cannot carry out DST, and $17 \%$ of prefectures have just gained the ability for DST in 2018-2019. The likelihood of insufficient personnel capacity and experience will affect the patient's diagnosis. Third, there are limitations on current drugresistance diagnostic algorithms regarding utilization of Xpert. Xpert is just a tool for the diagnosis of RR in bacteriologically confirmed TB in China and isn't used in the diagnosis of signs or symptoms of TB as recommended by the WHO to maximize the detection of TB and RR-TB cases [16]. Xpert is still an expensive tool for China because the number of people with presumptive TB symptoms in China is enormous (about 3 million people per year).

A third issue relates to the FQs susceptibility testing. Contrary to the increasing RR screening rate, the FQs testing proportion has been declining year by year from 2016 and only $41.0 \%$ of RR-TB cases were tested for FQs susceptibility in 2019. This was far lower than the $71 \%$ globally [2], and was lower than the $44 \%$ in South Africa [30]. According to WHO's good susceptibility testing coverage standards, although China's RR screening rate has exceeded $80 \%$, due to the low FQs susceptibility testing rate, China has not yet reached good testing coverage [2].
The reason why the ratio of rifampicin and FQs drug susceptibility testing showed opposite trends may be related to the fact that rifampicin testing increasingly uses molecular testing tools and can be conveniently implemented in county-level hospitals, hence its increased usage. FQs drug susceptibility testing is carried out at the prefecture level, and because traditional drug susceptibility methods can simultaneously obtain drug susceptibility results of other drugs, FQs drug resistance testing in China currently relies more on traditional methods. These factors may have contributed to the difference in the ratio of resistance testing of the two drugs. Another reason for the low proportion of FQs susceptibility testing is that the FQs screening rate is not a target indicator for the 13th Five-Year TB Program. Despite testing of FQs susceptibility, the result was not registered in the TBIMS in time. In addition, similar to the reason for the lower RR-TB detection, the DST capability of some newly implemented PMDT prefectures, especially second-line drug susceptibility testing, still need to be improved. In addition to the above reasons, since FQs susceptibility testing is currently not free, this also leads to a lower proportion of FQs testing.

FQs are the backbone of RR treatment regimens and FQs resistance is associated with poor treatment outcomes. WHO recommended that, before initiating treatment for RR-TB cases, it is necessary to carry out susceptibility testing for FQs, preferably by rapid assay [31]. The resistance rate of FQs among RR-TB patients in China is $27.4 \%$ [32], which is higher than the global average of $21 \%$ [2]. This implies that at least $1 / 4$ of RR-TB cases enrolled for treatment that have not undergone FQs susceptibility testing will have a risk of poor treatment outcomes. China has introduced the short-course 9-12 month chemotherapy regimen recommended by the WHO. The key eligible criterion for using the shortterm regimen is that the patient is not resistant to FQs. Therefore, in order to enable patients to apply appropriate chemotherapy regimens to ensure a therapeutic effect, it is imperative to conduct FQs susceptibility testing for RR-TB patients.

There are some limitations in our study. We used data from the TBIMS. The registration system only classifies the RR testing as "fast" and "traditional", and it is not yet possible to distinguish whether the FQs susceptibility testing is "molecular" or "traditional". If we need to understand the current ability of FQs rapid molecular DST in China, a special investigation needs to be conducted. In addition, the current system is unable to obtain data about whether the results of RR are directly diagnosed at the county level or by transporting specimens to prefectures for diagnosis. This data is meaningful for the national budget for the 
delivery of sputum specimens and the layout of rifampicin resistance testing institutions.

\section{Conclusions}

Our analysis demonstrated that China's political commitment together with aggressive 13th Five-Year TB Program targets, have had a great impact on the detection of RR-TB cases in China. After the change in RR-TB detection strategy, it exhibited a significant increase in the screening rate of RR and the number of RR-TB cases. At present, screening of RR is routine work for NTP. Nonetheless, there is still a gap in the detection of RR-TB cases. FQs susceptibility testing is another key concern in China's current DST. In order to achieve the goal of END TB, China also needs to reduce the spread of $\mathrm{RR}-\mathrm{TB}$ and effectively strengthen the laboratory capability at the county-level to allocate health resources reasonably.

\section{Abbreviations}

RR-TB: Rifampicin resistance tuberculosis; PMDT: Programmatic Management of Drug-resistant Tuberculosis; DST: Drug susceptibility testing; TBIMS: Tuberculosis Information Management System; FQs: Fluoroquinolones; TB:Tuberculosis; WHO: World Health Organization; 13th Five-Year TB Program: 13th Five-Year National Tuberculosis Prevention and Control Program (2016-2020); Xpert: Xpert MTB/RIF; LPAs: Line probe assays; MeltPro: MeltPro TB assay; NTP: National TB Program; NTM: Non-tuberculous mycobacteria.
\end{abstract}

\section{Acknowledgements}

Not applicable.

\section{Authors' contributions}

SW, LR conceptualized the paper, SW drafted the manuscript, LR reviewed the paper, and RY participated in the data interpretation and discussion. $L T$ DX, JJ participated in data analysis. All authors read and approved the final manuscript.

\section{Funding}

This study was supported by China CDC-Lilly Foundation Multidrug-resistant Tuberculosis Prevention and Control Project (Lilly Foundation Grant ID \# 16854).

\section{Availability of data and materials}

All data during this study are included in this published article.

\section{Declarations}

Ethics approval and consent to participate

Our analysis is based on data from the national TBIMS and no ethical approval is required.

\section{Consent for publication \\ Not applicable.}

\section{Competing interests}

The authors declare no competing interests.

Received: 4 May 2021 Accepted: 5 July 2021

Published online: 17 July 2021

\section{References}

1. World Health Organization. Definitions and reporting framework for tuberculosis-2013 revision. WHO/HTM/TB/2013.2. https://www.who.int/ publications/i/item/9789241505345. Accessed 25 June 2021.

2. World Health Organization. Global tuberculosis report 2020. Geneva: WHO; 2020. https://www.who.int/publications/i/item/9789240013131. Accessed 25 June 2021

3. Wang L, Li R, Xu C, Zhang H, Ruan Y, Chen M, et al. The Global Fund in China: multidrug-resistant tuberculosis nationwide programmatic scaleup and challenges to transition to full country ownership. PLoS One. 2017;12(6):e0177536.

4. Chen M, Li R, Ruan Y, Xu C. Global Fund MDR-TB control project in China-achievements and experience. Beijing: People's Medical Publishing House; 2015. (In Chinese).

5. Xu C, Li R, Shewade HD, Jeyashree K, Ruan Y, Zhang C, et al. Attrition and delays before treatment initiation among patients with MDR-TB in China (2006-13): magnitude and risk factors. PLoS One. 2019;14(4):e0214943.

6. World Health Organization. Global tuberculosis report 2013. Geneva: WHO; 2013. https://www.who.int/publications/i/item/9789241564656. Accessed 25 June 2021.

7. World Health Organization. Global tuberculosis report 2014. Geneva: WHO; 2014. https://www.who.int/publications/i/item/9789241564809. Accessed 25 Jun e2021.

8. World Health Organization. Global tuberculosis report 2015. Geneva: WHO; 2015. https://www.who.int/publications/i/item/9789241565059. Accessed 25 June 2021.

9. World Health Organization. The end TB strategy. Geneva: WHO; 2014. https://www.who.int/publications/i/item/WHO-HTM-TB-2015.19. Accessed 25 June 2021.

10. "13th Five-Year" National Tuberculosis Prevention and Control Plan. http://www.gov.cn/zhengce/content/2017-02/16/content_5168491.htm. Accessed 8 June 2021. (In Chinese).

11. World Health Organization. Global tuberculosis report 2019. Geneva: WHO; 2019. https://www.who.int/publications/i/item/global-tuberculos is-report-2019. Accessed 25 June 2021.

12. Action Plan to Stop Tuberculosis (2019-2022). http://www.nhc.gov.cn/jkj/ s3589/201906/b30ae2842c5e4c9ea2f9d5557ad4b95f.shtml. Accessed 8 June 2021. (In Chinese).

13. Huang F, Cheng S, Du X, Chen W, Scano F, Falzon D, et al. Electronic recording and reporting system for tuberculosis in China: experience and opportunities. J Am Med Inform Assoc. 2014;21(5):938-41.

14. The Collaborative Group for the Meta-Analysis of Individual Patient Data in MDR-TB treatment-2017. Treatment correlates of successful outcomes in pulmonary multidrug-resistant tuberculosis: an individual patient data meta-analysis. Lancet. 2018;392(10150):821-34.

15. WHO consolidated guidelines on tuberculosis. Module 4: Treatment. Drug-resistant tuberculosis treatment. Geneva: World Health Organization; 2020. https://www.who.int/publications/i/item/9789240007048. Accessed 25 June 2021.

16. WHO consolidated guidelines on tuberculosis. Module 3: Diagnosis. Rapid diagnostics for tuberculosis detection. Geneva: World Health Organization; 2020. https://www.who.int/publications/i/item/who-conso lidated-guidelines-on-tuberculosis-module-3-diagnosis---rapid-diagn ostics-for-tuberculosis-detection. Accessed 25 June 2021.

17. Pang $Y$, Dong $H$, Tan $Y$, Deng $Y$, Cai $X$, Jing H, et al. Rapid diagnosis of MDR and XDR tuberculosis with the MeltPro TB assay in China. Sci Rep. 2016;6:25330.

18. Pang Y, Xia H, Zhang Z, Li J, Dong Y, Li Q, et al. Multicenter evaluation of genechip for detection of multidrug-resistant Mycobacterium tuberculosis. J Clin Microbiol. 2013;51(6):1707-13.

19. Zhao YL. Quality assurance manual for TB laboratory. Beijing: People's Medical Publishing House; 2017. (In Chinese).

20. National Health Commission of the People's Republic of China. Classification of tuberculosis. WS196 - 2017. http://www.nhc.gov.cn/ewebeditor/ uploadfile/2017/12/201712. Accessed 8 June 2021. (In Chinese).

21. Wang Y. Guidelines for the prevention and control of multidrug-resistant tuberculosis. Beijing: Military Science Publishing House; 2012. (In Chinese).

22. Harris RC, Grandjean L, Martin LJ, Miller AJ, Nkang JE, Allen V, et al. The effect of early versus late treatment initiation after diagnosis on the 
outcomes of patients treated for multidrug-resistant tuberculosis: a systematic review. BMC Infect Dis. 2016;16(1):193.

23. Cox H, Dickson-Hall L, Ndjeka N, Van't Hoog A, Grant A, et al. Delays and loss to follow-up before treatment of drug-resistant tuberculosis following implementation of Xpert MTB/RIF in South Africa: a retrospective cohort study. PLoS Med. 2017;14(2):e1002238.

24. Zürcher K, Ballif M, Fenner L, Borrell S, Keller PM, Gnokoro J, et al. Drug susceptibility testing and mortality in patients treated for tuberculosis in high-burden countries: a multicentre cohort study. Lancet Infect Dis. 2019;19(3):298-307.

25. Jeon D, Kang H, Kwon YS, Yim JJ, Shim TS. Impact of molecular drug susceptibility testing on the time to multidrug-resistant tuberculosis treatment initiation. J Korean Med Sci. 2020;35(35):e284.

26. Wu Z, Rueda ZV, Li T, Zhang Z, Jiang Y, Sha W, et al. Effect of the Xpert MTB/RIF on the detection of pulmonary tuberculosis cases and rifampicin resistance in Shanghai, China. BMC Infect Dis. 2020;20(1):153.

27. Li X, Xu C, Wei S, Hu D, Liu X, Zhang H. Analysis of tuberculosis laboratory capacity building in China from 2011 to 2015. Chin J Public Health Mang. 2019;35(4):441-4 (In Chinese).

28. Yang C, Luo T, Shen X, et al. Transmission of multidrug-resistant Mycobacterium tuberculosis in Shanghai, China: a retrospective observational study using whole-genome sequencing and epidemiological investigation. Lancet Infect Dis. 2017;17:275-84.

29. Ge E, Li D, Luo M, Tsui KWS, Waye MMY, Shen X, Wei X. Transmission of multidrug-resistant tuberculosis in Shanghai: roles of residential status. Int J Tuberc Lung Dis. 2018;22(12):1462-8.

30. Jacobson KR, Barnard M, Kleinman MB, et al. Implications of failure to routinely diagnose resistance to second-line drugs in patients with rifampicin-resistant tuberculosis on Xpert MTB/RIF: a multisite observational study. Clin Infect Dis. 2017:64(11):1502-8.

31. WHO operational handbook on tuberculosis. Module 4: Treatment. Drug-resistant tuberculosis treatment. Geneva: World Health Organization; 2020. https://www.who.int/publications/i/item/9789240006997. Accessed 25 June 2021

32. National Baseline Investigation Report on Tuberculosis Drug Resistance (2007-2008). Edited by the former Ministry of Health of the People's Republic of China. Beijing: People"s Medical Publishing House. 2010. (In (hinese).
Ready to submit your research? Choose BMC and benefit from:

- fast, convenient online submission

- thorough peer review by experienced researchers in your field

- rapid publication on acceptance

- support for research data, including large and complex data types

- gold Open Access which fosters wider collaboration and increased citations

- maximum visibility for your research: over $100 \mathrm{M}$ website views per year

At BMC, research is always in progress.

Learn more biomedcentral.com/submissions 